\title{
Migration-driven aggregate behaviors of human mesenchymal stem cells on a dendrimer-immobilized surface direct differentiation toward a cardiomyogenic fate commitment
}

\author{
Yuuki Ogawa, Mee-Hae Kim, and Masahiro Kino-oka* \\ Department of Biotechnology, Graduate School of Engineering, Osaka University, 2-1 Yamadaoka, \\ Suita, Osaka 565-0871, Japan
}

*Corresponding author.

E-mail: kino-oka@bio.eng.osaka-u.ac.jp

Tel: +81-(0)6-6879-7444

Fax: $+81-(0) 6-6879-4246$

Running title: CARDIOMYOGENIC FATE IN AGGREGATE MIGRATORY BEHAVIOR

Footnote: Much of this work forms the basis of the Ph.D. dissertation of Yuuki Ogawa 


\section{ABSTRACT}

Dynamic behaviors of cell aggregates on a dendrimer surface were investigated to drive the

3 directed differentiation of human mesenchymal stem cells (hMSCs) toward a cardiomyogenic

4 lineage. Cell aggregates on the polyamidoamine dendrimer surface with fifth-generation (G5) of

5 dendron structure showed dynamic changes in morphology associated with repetitive stretching and

6 contracting during migration. Spatial-temporal observations revealed cellular movement in single

7 aggregates by their morphological change through stretching and contracting on the G5 surface,

8 suggesting that the dynamic behavior of aggregate causes mixing of cells. However, aggregates

9 without cell-substrate adhesions on the low-binding culture surface sustained their spherical

10 morphology without cellular movement within a single aggregate. Furthermore, $\beta$-catenin was

11 observed at nuclei in aggregates on the G5 surface, and expression of the cardiomyocyte marker

12 cardiac Troponin $\mathrm{T}(\mathrm{cTnT})$ was detected. However, $\beta$-catenin localized to the nuclei only in the

13 outer region of the aggregate on the low-binding culture surface, and cTnT expression was

14 restricted at the exterior surface of the aggregates. These observations indicate that cell mixing

15 within aggregates on the G5 surface induced the directed differentiation of hMSCs toward a

16 cardiomyogenic lineage by nuclear translocation of $\beta$-catenin through dissociation of cell-cell

17 adhesions. These results suggest that migration-driven aggregate behaviors on the dendrimer

18 surface caused repeated morphological changes of aggregate through stretching and contracting,

19 leading to the directed differentiation of hMSCs toward a cardiomyogenic fate commitment.

21 [Key words: migration-driven aggregate behaviors, dendrimer surface, cell-cell adhesion, 22 cardiomyogenic lineage, human mesenchymal stem cells] 


\section{INTRODUCTION}

2 Human mesenchymal stem cells (hMSCs) can differentiate into several cell types, such as

3 chondrocytes, adipocytes, osteoblasts, neurons, and cardiomyocytes (1-3). Uncommitted hMSCs

4 have extensive proliferative ability in culture while retaining their multilineage differentiation

5 potential, which makes them attractive candidates for cell-based therapeutic approaches (4).

6 However, the low differentiation efficiency and poor reproducibility of hMSCs is a major limitation

7 to their clinical use.

8 To establish cellular microenvironments that promote differentiation into objective cell types,

9 many researchers have focused on exogenous stimulants such as growth factors and extracellular

10 matrices to mimic the native microenvironment (2-5). Transforming growth factor- $\beta$ (TGF- $\beta$ )

11 superfamily members direct differentiation of hMSCs toward a specific lineage: Mohanty et al. (5)

12 reported that TGF- $\beta 1$ contributes to hMSC differentiation into a cardiomyogenic lineage, showing

$1321 \%$ cardiomyocyte-like cells. An alternative approach to hMSC differentiation involves endogenous

14 stimulation by regulation of cell behaviors (6-8). The endogenous Rho GTPase family includes

15 RhoA, Rac1, and Cdc42 and regulates the assembly and disassembly of actin cytoskeleton, thereby

16 leading to changes in cellular behaviors such as cell migration (9-12). Rac1 activation is required to

17 promote actin polymerization for cell extension, whereas RhoA activity regulates the formation of

18 actin stress fibers and is antagonistic toward Rac1 activity (10,11). Activation of Rac1 induces

19 differentiation of hMSCs into the myogenic lineage, and its inactivation induces differentiation into

20 the chondrogenic lineage (6). Thus, endogenous Rho family GTPase signaling pathways are

21 considered to play a central role in regulation of cell fate decisions, and the fate choice relies on

22 modulation of the network of transcription factors. Furthermore, stimulation of cell-cell adhesion by

23 aggregate culture has been shown to facilitate hMSC differentiation (13), suggesting that aggregate

24 culture and intercellular communication play significant roles in regulating cell differentiation.

25 In our previous studies (14-16), we demonstrated that formation of hMSC aggregates on the 
1 dendrimer surface through active migration with morphological changes led to directed

2 differentiation of hMSCs toward a cardiomyogenic fate commitment. Repetitive collapse and

3 re-formation of the aggregates increased the population of cardiomyocyte-like cells by passage

4 culture of aggregates on a new dendrimer surface (15). In this study, we investigated the migratory

5 behaviors of hMSC aggregates on the dendrimer surface to enhance directed differentiation toward a

6 cardiomyogenic lineage. Based on examination of aggregate migratory behaviors on the dendrimer

7 surface, we discuss the fundamental mechanisms of cell-cell and cell-substrate adhesion in relation

8 to guidance of cell fate.

\section{MATERIALS AND METHODS}

$10 \quad$ Cells and culture conditions

11 Bone marrow-derived hMSCs (Lot no. 0000183402; Lonza, Walkersville, MD, USA) were used

12 in the following experiments. Routine subcultures were conducted as described previously (16). For

13 all experiments, hMSCs were harvested in Dulbecco's modified Eagle's medium (DMEM;

14 Sigma-Aldrich, St. Louis, MO, USA) supplemented with $10 \%$ fetal bovine serum and antibiotics

15 (Life Technologies, Carlsbad, CA, USA). The seeding density was $5.0 \times 10^{3}$ cells $/ \mathrm{cm}^{2}$, and medium 16 changes were performed every 3 days.

17 hMSCs were exposed to medium with $50 \mu \mathrm{g} / \mathrm{mL}$ Rac1 activator HMG-1 (Sigma-Aldrich) or 5

$18 \mu \mathrm{g} / \mathrm{mL}$ Rac1 inhibitor NSC23766 (Calbiochem, Merck, Darmstadt, Germany) from day 3 to day 10

19 to activate or inhibit migratory behaviors of cell aggregates, respectively.

20 Preparation of dendrimer-immobilized surface

21 The fifth-generation dendrimer surface (G5 surface) was prepared on the tissue-cultured 22 polystyrene (PS) surface of a square 8-well plate (Nunc, Roskilde, Denmark) and $35 \mathrm{~mm}$ culture dish

23 (Ibidi GmbH, Martinsried, Germany) as described previously (16). Briefly, hydroxyl groups on the 24 starting materials were displayed by addition of potassium tert-butoxide. Then, dendron structures 
were constructed by alternately adding glutaraldehyde and Tris(2-aminoethyl)amine solution ( $\mathrm{pH} 9.0$,

2 adjusted with $0.1 \mathrm{~mol} / \mathrm{L} \mathrm{NaOH}$ ), respectively, five times. D-glucose was displayed as a terminal 3 ligand.

\section{Time-lapse observations}

Time-lapse observations were carried out by obtaining images every $10 \mathrm{~min}$ at several positions using an observation tool (BioStudio-T; Nikon Engineering, Kanagawa, Japan).

To track individual nuclei within the aggregates, the cells were transfected with nucleus-green fluorescent protein (GFP) by baculovirus (CellLight nucleus-GFP; Life Technologies) according to

9 the commercially available protocol. There was no significant difference in migratory behavior and

10 differentiation between cultures of transfected cells and untransfected cells; therefore, transfected

11 cells were not isolated from untransfected cells. Cells were then stained with a cytoplasmic dye

12 (CellTracker ${ }^{\mathrm{TM}}$ Orange CMTMR; Life Technologies) used to distinguish hMSC aggregate behavior 13 according to the commercially available protocol. Images were obtained every 20 min using a 14 time-lapse confocal laser scanning microscope (FV-10i; Olympus, Tokyo, Japan) through a 60× 15 objective lens. Tracking imaging analysis of the migration of individual cells within the aggregate was performed using image processing software (Imaris; Bitplane AG, Zurich, Switzerland).

\section{Immunofluorescence staining}

18 Immunostaining was carried out as described previously (14). Briefly, cells were fixed with $4 \%$ 19 paraformaldehyde. Then, the cells were permeabilized by incubation in PBS with $0.5 \%$ 20 polyoxyethylene (10) octylphenyl ether. After masking non-specific proteins (Block Ace; Dainippon

21 Sumitomo Pharma Co., Ltd., Osaka, Japan), the cells were incubated with anti- $\beta$-catenin primary 22 antibody (Santa Cruz Biotechnology, Santa Cruz, CA, USA) or anti-cardiac Troponin T (cTnT) 23 antibody (Abcam, Cambridge, UK). The cells were reacted with secondary antibody (Alexa Flour 24 488-conjugated anti-mouse IgG; Life Technologies). Nuclei and F-actin were stained with 25 4',6-diamidino-2-phenylindole (DAPI) and rhodamine phalloidin (Life Technologies) respectively. 
1 Images were obtained using a confocal laser scanning microscope (FV-1000; Olympus) through a

$260 \times$ objective lens.

3 Determination of the cardiomyogenic potential of aggregates

4 Quantitative assessment of cardiomyogenic differentiation was conducted as described

5 previously (15). Briefly, cells incubated for a specified number of days were collected by enzymatic

6 treatment and re-seeded on the PS surface. The cells were stained using cTnT antibody as above. The

7 ratio of cTnT-positive cell number to DAPI-positive cell number $\left(X_{\mathrm{P}} / X_{\mathrm{T}}\right)$ was calculated.

$8 \quad$ Statistical analysis

9 All experiments were performed at least three times, and data are expressed as means with 10 standard deviations. Student's $t$-test and the Tukey-Kramer method were used to determine statistical 11 significances among the data sets; $p$-values less than 0.01 were considered significant.

\section{RESULTS}

13 Dynamic behaviors of hMSC aggregates

14 To understand the behavioral changes in hMSC aggregates, we performed time-lapse imaging 15 of representative aggregates cultured on the G5 surface and low-binding culture surface. The cells on 16 the G5 surface showed repetitive morphological changes through stretching and contracting during

17 migration, and their migratory behaviors were repeated for cultivation from day 7 to day 10 (Movie

$18 \mathrm{~S} 1)$. However, cells on the low-binding culture surface formed ball-like cell aggregates and 19 maintained this shape over the entire observation period (Movie S2).

20 To observe the movement of nuclei within a single aggregate during migration, the 21 nucleus-GFP-transfected hMSC aggregates were labeled with cell-tracker dye, and time-lapse series 22 were acquired using a confocal fluorescence microscope at day 8. On the G5 surface, active cell 23 migration was observed in cells located at the periphery of the aggregates, and cell migration leading 24 to the stretching and contracting of aggregates was observed (Fig. 1, Movie S3, and Movie S4). The Fig. 1 
nuclear position of cells attached to the surface (marked by a cross in each image) moved toward the upper side of the aggregate during contraction, indicating nuclear mixing within the aggregate.

3 However, on the low-binding culture surface, aggregates maintained their spherical morphology

4 without any drastic change in nuclear position (Fig. 1 and Movie S5). These results considered that

5 the dramatic change in aggregate morphology through active migration on the G5 surface caused

6 mixing of cells in aggregate.

$7 \quad \beta$-catenin translocation into nucleus and cTnT-based cardiomyogenic potential

8 Translocation of $\beta$-catenin into the nucleus and cardiomyogenic potential were observed by 9 immunostaining the hMSC aggregates for $\beta$-catenin and cTnT on the G5 surface and low-binding 10 culture surface at day 8 . Punctate $\beta$-catenin staining was observed at contact sites between adjacent 11 cells irrespective of the surface (Fig. 2). Comparison $\beta$-catenin nuclear translocation within Fig. 2

12 aggregates revealed its distribution in the nucleus throughout the entire aggregate from central to 13 peripheral regions on the G5 surface, whereas it was detected in the nucleus only at the peripheral 14 region on the low-binding culture surface (Fig. 2A and Fig. 2B).

15 Similarly, cTnT was strongly detected throughout the entire aggregate on the G5 surface at day 168 (Fig. 3A) but only in the peripheral region of the aggregate on the low-binding culture surface. The Fig. 3

17 quantitative estimation of the cardiomyogenic potential was calculated by the ratio of cTnT positive 18 cells $\left(X_{\mathrm{P}} / X_{\mathrm{T}}\right)$ in culture of hMSCs on both surfaces. As shown in Fig. S1, the $X_{\mathrm{P}} / X_{\mathrm{T}}$ value in hMSCs 19 cultured on the G5 surface was 2.2-fold higher than that on the low-binding culture surface.

\section{Effect of migratory behaviors on cardiomyogenic potential of hMSC aggregates}

21 The effect of migratory behaviors on the cardiomyogenic potential of hMSCs was studied by 22 exposure to medium with $50 \mu \mathrm{g} / \mathrm{mL}$ Rac1 activator or $5 \mu \mathrm{g} / \mathrm{mL}$ Rac1 inhibitor from day 3 to day 10 , 23 and mRNA expression of Rac1 was examined at days 8 and 10 in hMSCs cultured under these 24 conditions. The expression levels of Rac1 in hMSCs cultured on the G5 surface were significantly 25 increased or decreased with exposure to Rac1 activator and Rac1 inhibitor both at days 8 and 10 , 
compared to those without exposure (Fig. S2).

2 In cultures exposed to Rac1 activator, the aggregates on the G5 surface maintained dynamic

3 morphological changes through stretching and contracting from day 7 to day 10 (Movie S6),

4 although the morphological change of aggregate on the G5 surface without exposure decreased from

5 day 7 to day 10. In addition, Rac 1 activator-exposed cells exhibited mixing of cells in the aggregate

6 at days 8 and 10 through active migration of cultured cells attached to a surface (Movie S7 and

7 Movie S8), although the movement was suppressed at day 10 in culture without exposure (Movie S9).

8 This migratory pattern of nuclei within the aggregate mimicked that in unexposed cells at day 8 . In

9 contrast, incubation with the Rac1 inhibitor led to a flattened and well-spread aggregate morphology

10 without any spontaneous periodic change (Movie S10). The coordinated movement was suppressed

11 at days 8 and 10, which are correlated with suppression of the active migration of leader cells to

12 surface (Movie S11 and Movie S12).

13 The cardiomyogenic potential, the $X_{\mathrm{P}} / X_{\mathrm{T}}$ value was calculated in culture of hMSCs on the G5

14 surface with Rac1 activator or Rac1 inhibitor from day 3 to day 10. As shown in Fig. 4, the $X_{\mathrm{P}} / X_{\mathrm{T}}$ Fig. 4

15 value with Rac1 activator was higher than that without Rac1 activator at both days 8 and 10 . In

16 addition, the $X_{\mathrm{P}} / X_{\mathrm{T}}$ value of Rac1 activator-exposed cells at day 10 was higher than that at day 8 .

17 However, the $X_{\mathrm{P}} / X_{\mathrm{T}}$ values of Rac1 inhibitor-exposed cells both at days 8 and 10 were lower than

18 that of unexposed cells. In addition, the $X_{\mathrm{P}} / X_{\mathrm{T}}$ values for aggregates cultured on the low-binding

19 culture surface with Rac1 activator or Rac1 inhibitor were evaluated. In contrast to hMSC aggregates

20 on the G5 surface, the $X_{\mathrm{P}} / X_{\mathrm{T}}$ values were the same as those derived from unexposed cells at day 10 ,

21 regardless of culture conditions (Fig. S3).

DISCUSSION 
The formation of aggregates is an important event influencing the fate of cultured stem cells,

2 and the mechanisms of aggregate migratory behaviors need to be elucidated to understand cellular

3 differentiation. In this study, we investigated their effects on the directed differentiation of hMSCs

4 toward a cardiomyogenic fate commitment accompanied by dynamic migration behaviors within

5 the aggregates on the G5 surface (Fig. S4). The main feature of aggregate migration behavior is the

6 emergence of leader cells and their subsequent migration that is accompanied by nearby cells called

7 follower cells, thereby causing collective migration. Time-lapse observation (Movie S1) revealed

8 that aggregates grown on the G5 surface actively migrated and showed morphological fluctuations

9 with repetitive stretching and contracting. In addition, time-lapse fluorescent observation of nuclei

10 (Movie S2) revealed that dynamic change of aggregate morphology on the G5 surface caused the

11 cellular mixing in aggregate. However, aggregates without cell-substrate adhesion on the

12 low-binding culture surface maintained their morphology without any drastic change of nuclear

13 location within a single aggregate (Movie S5). In the analysis of lineage commitment of hMSCs

14 toward a cardiomyogenic fate, the expression of cTnT was prominently seen throughout aggregates

15 on the G5 surface but was exclusively located around the aggregates on the low-binding culture

16 surface (Fig. 2 and Fig. 3). For further enhancement of directed differentiation of hMSCs toward a

17 cardiomyogenic lineage, we applied the Rac1 activator and Rac1 inhibitor to regulate the migratory

18 behaviors of the aggregates on the G5 surface. In culture, exposure to the Rac1 activator caused

19 repetitive morphological changes through stretching and contracting during active migration of

20 cells on the G5 surface and enhanced directed differentiation of hMSCs toward a cardiomyogenic

21 lineage (Movie S6 and Fig. 4). The difference in migratory behavior of cell aggregates resulted in

22 varied properties between attached aggregates and unattached, floating aggregates. The dramatic

23 morphological change of aggregates through active migration on the G5 surface can lead to

24 coordinated movement (passive) of individual cells within the aggregate. Migration determines the

25 balance between cell contractile forces and cell-substrate adhesion through the rearrangement of 
1 integrin-mediated cell adhesion; thus, cell contractions can cause a secondary wave of

2 mechano-regulated outside-in and internal cell signal changes that control cell fate decisions (17).

3 Taken together, these findings revealed that the coordinated movement of cells driven by cell

4 adhesion to the G5 surface led to directed differentiation of hMSCs toward a cardiomyogenic fate

5 commitment.

Migration-driven aggregate behaviors direct differentiation toward a cardiomyogenic

7 fate commitment through cell-cell dissociation

8 The spontaneous formation and migratory behaviors of aggregates affect cell-substrate and

9 cell-cell adhesions within aggregates and thereby regulate cell differentiation signaling $(14,15)$.

10 Within migrating aggregates, cell-cell and cell-substrate adhesions are integrated so that cells

11 maintain interactions with neighboring cells and the underlying substratum. The coordinated

12 organization of cadherin-based and integrin-based adhesion complexes is required for coordinated

13 movement of large groups of cells, and spatiotemporal regulation of Rho family GTPases and actin

14 dynamics is required for collective cell migration (17-20). These mechanisms are dependent on the

15 synthesis and degradation of the extracellular matrix (7). Our previous reports indicated that the

16 dynamic cellular behaviors on the G5 surface were changed due to the adsorption and assembly of

17 fibronectin (16). On the G5 surface, adsorption of fibronectin induces the extension of cells, and

18 that fibronectin assembly affected by matrix metalloproteinases plays important roles in the

19 stabilization of focal adhesions associated with cytoskeletal formations. Because paxillin

20 phosphorylation enhances the turnover of focal adhesion with lamellipodium protrusion, focal

21 adhesion turnover is thought to be promoted by active cell migration through paxillin

22 phosphorylation (16). In addition, cadherins at cell-cell adhesion sites may show responses to

23 mechanical signals similar to paxillin at focal adhesions, which is supported by the similarity of

24 interactions in cell-cell and cell-substrate adhesion structures with the actin cytoskeleton $(16,18)$.

25 These results suggest that extracellular signals can be locally integrated by the endogenous Rho 
1 family GTPases to coordinate the dynamic cytoskeletal rearrangements and cell-cell

2 communication necessary for directing stem cell fates. The $\beta$-catenin plays important roles in stem

3 cell differentiation as a transcription factor and cell-cell adhesion by connecting to N-cadherin.

4 Dynamic changes in $\beta$-catenin expression and transcriptional activity were regulated in response to

5 altered cell adhesion during migration. Consistent with previous results (15), cell aggregates on the

6 G5 surface induced cTnT expression due to the morphological changes, and the results from this

7 study suggest that $\beta$-catenin signaling in response to changes in migration behaviors of hMSC

8 aggregates may be implicated in the guidance of cardiomyogenic fate. $\beta$-catenin was expressed at

9 the interface among cells in the aggregates on the G5 surface and the low-binding culture surface.

10 In addition, on the G5 surface, $\beta$-catenin at the nuclei and cTnT were observed throughout the

11 aggregates, whereas they were only detected at the outer layer of the aggregates on the low-binding

12 culture surface (Fig. 2 and Fig. 3). The changes in $\beta$-catenin transcriptional activity with

13 relocalization of the position of each cell within the aggregate, which made dynamic morphological

14 changes on the G5 surface combined with changes in $\mathrm{N}$-cadherin expression indicate that cell

15 association during aggregate migration can affect subsequent pathway activation and differentiation.

16 It is likely that similar interrelated signaling between cadherins and $\beta$-catenin may enable the pool

17 of cadherin-bound $\beta$-catenin to become available for nuclear translocation and signaling upon

18 remodeling of cadherins during active migration of aggregates. The cytoplasmic stabilization of

19 dephosphorylated $\beta$-catenin permits the translocation of $\beta$-catenin to the nucleus, where it acts as a

20 transcriptional co-activator with T-cell factor/lymphoid enhancer-binding factor to regulate the

21 transcription of target genes during stem cell differentiation (21-25). Therefore, the morphological

22 changes in aggregates due to adsorption and assembly of fibronectin on the G5 surface led to the

23 dissociation of cell-cell adhesion, introducing to the directed differentiation toward a

24 cardiomyogenic lineage through transcriptional activity of $\beta$-catenin.

25 In conclusion, the present study indicates that the migratory behaviors of aggregates on the G5 
1 surface associated with morphological changes in the aggregates through stretching and contracting

2 induce directed differentiation of hMSCs toward a cardiomyogenic fate commitment. The active

3 migration of cells on the G5 surface leads to coordinated movement of cells within the aggregates

4 during stretching and contracting. We found that the nuclear translocation of $\beta$-catenin due to the

5 cell movement within the aggregate plays important roles in the directed differentiation of hMSCs

6 toward a cardiomyogenic fate commitment. These results suggest that the G5 surface offers a

7 model for substrate design based on aggregate migratory behaviors as a tool for driving a

8 cardiomyogenic lineage specification in an ex vivo stem cell culture system.

\section{ACKNOWLEDGMENTS}

10 This work was partially supported by the Japan Science and Technology Agency (JST), 11 Grants-in-Aid for Scientific Research (B) (no. 21360402) from the Ministry of Education, Culture,

12 Sports, Science and Technology of Japan, by the MEXT project, "Creating Hybrid Organs of the 13 future" at Osaka University, and by the Japan Society for the Promotion of Science (JSPS) through 14 Japanese-German Graduate Externship. 
2 1. Bieback, K., Wuchter, P., Besser, D., Franke, W., Becker, M., Ott, M., Pacher, M., Ma, N.,

3 Stamm, C., Klüter, H., Müller, A., and Ho, A. D.: Mesenchymal stromal cells (MSCs):

4 science and f(r)iction, J. Mol. Med. (Berl.), 90, 773-782 (2012).

5

6

2. Gimble, J. M., Guilak, F., Nuttall, M. E., Sathishkumar, S., Vidal, M., and Bunnell, B. A.: In vitro differentiation potential of mesenchymal stem cells, Transfus. Med. Hemother., 35, $228-238(2008)$.

3. Santiago, J. A., Pogemiller, R., and Ogle, B. M.: Heterogeneous differentiation of human mesenchymal stem cells in response to extended culture in extracellular matrices, Tissue Eng. Part A, 15, 3911-3922 (2009).

4. Oldershaw, R. A.: Cell sources for the regeneration of articular cartilage: the past, the horizon and the future, Int. J. Exp. Pathol., 93, 389-400 (2012).

5. Mohanty, S., Bose, S., Jain, K. G., Bhargava, B., and Airan, B.: TGF $\beta 1$ contributes to cardiomyogenic-like differentiation of human bone marrow mesenchymal stem cells, Int. J. Cardiol., 163, 93-99 (2013).

6. Gao, L., McBeath, R., and Chen, C. S.: Stem cell shape regulates a chondrogenic versus myogenic fate through Rac1 and N-cadherin. Stem Cells, 28, 564-572 (2010).

7. Lin, H., Yang, G., Tan, J., and Tuan, R. S.: Influence of decellularized matrix derived from human mesenchymal stem cells on their proliferation, migration and multi-lineage differentiation potential, Biomaterials, 33, 4480-4489 (2012).

8. Ridley, A. J.: Rho GTPases and actin dynamics in membrane protrusions and vesicle trafficking, Trends Cell Biol., 16, 522-529 (2006).

9. Ridley, A. J., Paterson, H. F., Johnston, C. L., Diekmann, D., and Hall, A.: The small GTP-binding protein rac regulates growth factor-induced membrane ruffling, Cell, 70, 401-410 (1992).

10. Flevaris, P., Stojanovic, A., Gong, H., Chishti, A., Welch, E., and Du, X.: A molecular 
switch that controls cell spreading and retraction, J. Cell Biol., 179, 553-565 (2007).

11. Pucéat, M., Travo, P., Quinn, M. T., and Fort, P.: A dual role of the GTPase Rac in cardiac differentiation of stem cells, Mol. Biol. Cell, 14, 2781-2792 (2003).

12. Sugimoto, K., Ohkawara, H., Nakamura, Y., Takuwa, Y., Ishibashi, T., and Takeishi, Y.: Receptor for advanced glycation end products - membrane type1 matrix metalloproteinase axis regulates tissue factor expression via RhoA and Rac1 activation in high-mobility group box-1 stimulated endothelial cells, PLoS One, 9, e114429 (2014).

13. Liu, B.-H., Yeh, H.-Y., Lin, Y.-C., Wang, M.-H., Chen, D. C., Lee, B.-H., and Hsu, S.-H.: Spheroid formation and enhanced cardiomyogenic potential of adipose-derived stem cells grown on chitosan, BioRes.Open Access, 2, 28-39 (2013).

14. Kim, M. H., Kino-oka, M., Maruyama, N., Saito, A., Sawa, Y., and Taya, M.: Cardiomyogenic induction of human mesenchymal stem cells by altered Rho family GTPase expression on dendrimer-immobilized surface with D-glucose display, Biomaterials, 31, 76667677 (2010).

15. Kim, M. H., Ogawa, Y., Yamada, K., Taya, M., and Kino-oka, M.: Directed differentiation of human mesenchymal stem cells toward a cardiomyogenic fate commitment through formation of cell aggregates, Biochem. Eng. J., 84, 53-58 (2014).

16. Ogawa, Y., Kim, M. H., and Kino-Oka, M.: Changes in human mesenchymal stem cell behaviors on dendrimer-immobilized surfaces due to mediation of fibronectin adsorption and assembly, J. Biosci. Bioeng., 120, 709-714 (2015).

17. Wang, C., Chowdhury, S., Driscoll, M., Parent, C. A., Gupta, S. K., and Losert, W.: The interplay of cell-cell and cell-substrate adhesion in collective cell migration, J. R. Soc. Interface, 11, 20140684 (2014).

18. Binamé, F., Pawlak, G., Roux, P., and Hibner, U.: What makes cells move: requirements and obstacles for spontaneous cell motility, Mol. Biosyst., 6, 648-661 (2010),

19. Vogel, V. and Sheetz, M. P.: Cell fate regulation by coupling mechanical cycles to biochemical 
signaling pathways, Curr. Opin. Cell Biol., 21, 38-46 (2009).

2 20. Petrie, R. J. and Yamada, K. M.: At the leading edge of three-dimensional cell migration, J.

$3 \quad$ Cell Sci., 125, 5917-5926 (2012).

4 21. Nelson, W. J. and Nusse, R.: Convergence of Wnt, beta-catenin, and cadherin pathways,

$5 \quad$ Science, 303, 1483-1487 (2004).

6 22. Arnsdorf, E. J., Tummala, P., and Jacobs, C. R.: Non-canonical Wnt signaling and $7 \quad \mathrm{~N}$-cadherin related beta-catenin signaling play a role in mechanically induced osteogenic cell 8 fate, PLoS One, 4, e5388 (2009).

9 23. Wu, X., Tu, X., Joeng, K. S., Hilton, M. J., Williams, D. A., and Long, F.: Rac1 activation 10 controls nuclear localization of beta-catenin during canonical Wnt signaling, Cell, 133, 340$11353(2008)$

12 24. Heo, J. S. and Lee, J. C.: $\beta$-catenin mediates cyclic strain-stimulated cardiomyogenesis in 13 mouse embryonic stem cells through ROS-dependent and intergrin-mediated PI3K/Akt 14 pathways, J. Cell Biochem., 112, 1880-1889 (2011).

15 25. Bai, X. L., Zhang, Q., Ye, L. Y., Liang, F., Sun, X., Chen, Y., Hu, Q. D., Fu, Q. H., Su, W., 16 Chen, Z., Zhuang, Z. P., and Liang, T. B.: Myocyte enhancer factor $2 \mathrm{C}$ regulation of 17 hepatocellular carcinoma via vascular endothelial growth factor and Wnt/ $\beta$-catenin signaling, 18 Oncogene, 34, 4089-4097 (2015). 


\section{FIGURE LEGENDS}

2 FIG. 1. Still images of time-lapse fluorescence observations of cytoplasm (red) and GFP-transfected nuclei (green) in hMSC aggregates cultured on the G5 surface (A-D) and low-binding culture surface (E and F) at day 8. The images show 3D-constructed images of aggregates. Panels A-D show the morphological fluctuations with repetitive stretching (A, B) and contracting (C, D) of aggregates grown on the G5 surface. Scale bars: $50 \mu \mathrm{m}$.

FIG. 2. Immunostaining of $\beta$-catenin (green) in hMSCs cultured on the G5 surface (A) and low-binding culture surface (B) at day 8. Cell nuclei were stained with DAPI (blue). Panels A and B show confocal images of $\beta$-catenin and nuclei in the middle sections of $x$-z stack images. Panels A1-B2 show enlarged images of the white boxed areas of panels A and B. Red arrows show the translocation of $\beta$-catenin into the nucleus. Scale bars: $50 \mu \mathrm{m}(\mathrm{A}, \mathrm{B})$ and $10 \mu \mathrm{m}(\mathrm{A} 1, \mathrm{~B} 2)$.

FIG. 3. Immunostaining of cTnT (green) in hMSCs cultured on the G5 surface (A) and low-binding culture surface (B) at day 8. Cell nuclei were stained with DAPI (blue). The confocal images of cTnT are from the middle section of the $x$-z stack images. Scale bars: $50 \mu \mathrm{m}$.

FIG. 4. Ratio of cTnT positive cells, $X_{\mathrm{P}} / X_{\mathrm{T}}$, in hMSCs on the G5 surface in culture conditions when exposed to medium without (A) and with Rac1 activator (B) and Rac1 inhibitor (C) from day 3 to day 10 by co-immunostaining with cTnT and DAPI after re-seeding to the PS surface by collecting all cells with enzymatic treatment at days 8 and 10. Open and closed bars show $X_{\mathrm{P}} / X_{\mathrm{T}}$ at days 8 and 10 , respectively. Vertical bars indicate the standard deviations $(n=3)$. Statistical significance was according to one-way analysis of variance (ANOVA) and Tukey-Kramer post-hoc test $\left({ }^{*} p<0.01\right)$. 
Fig 1

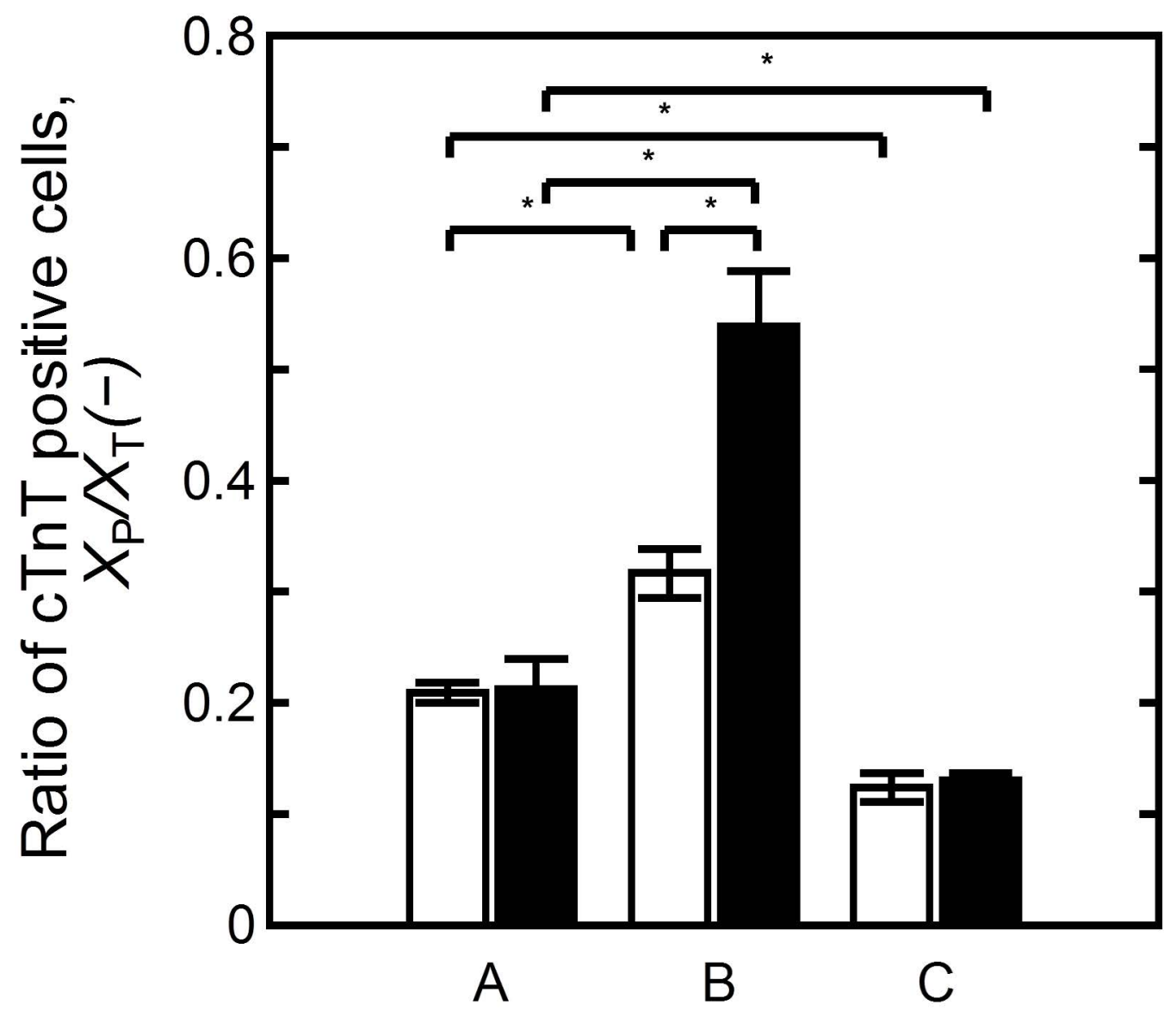


Fig 2

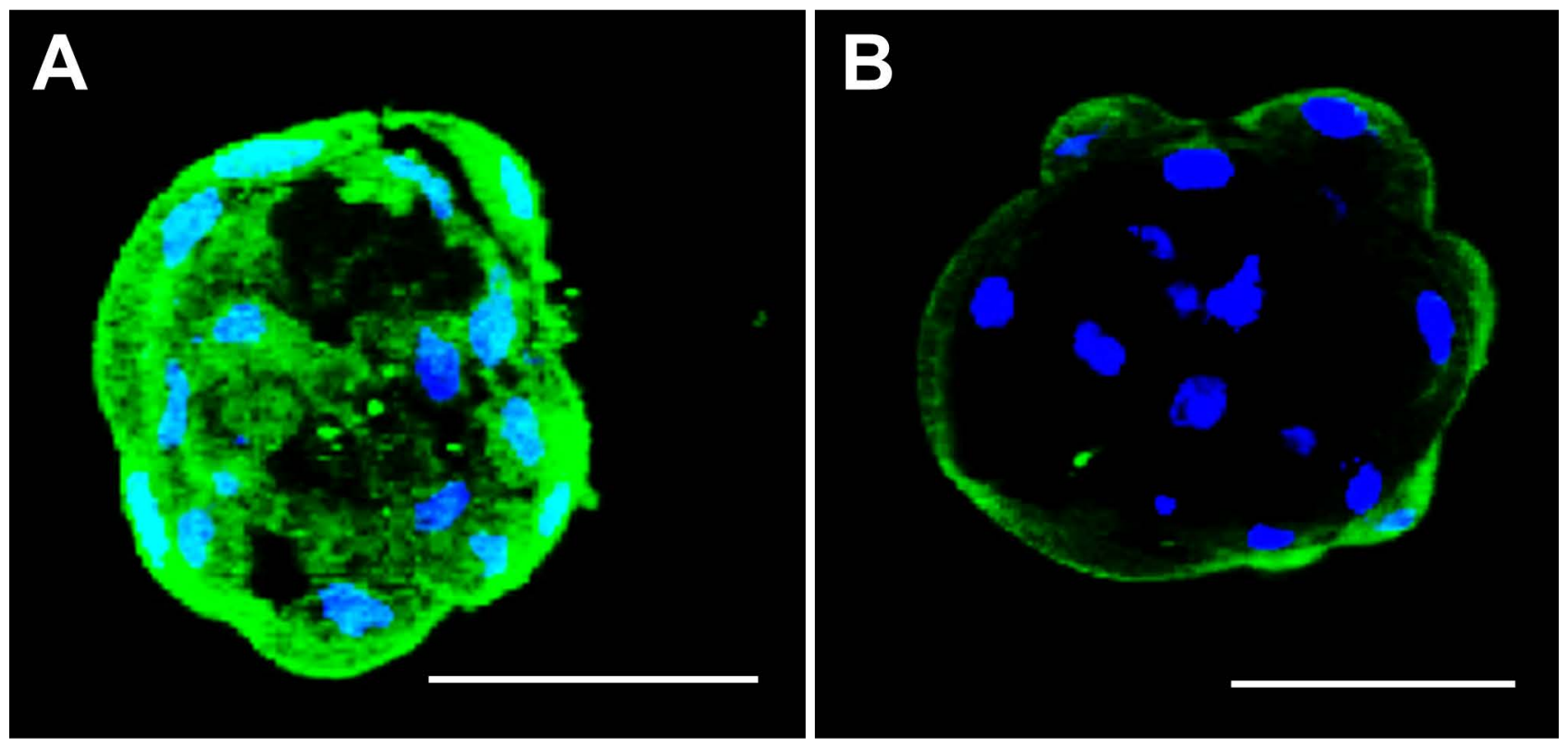


Fig 3
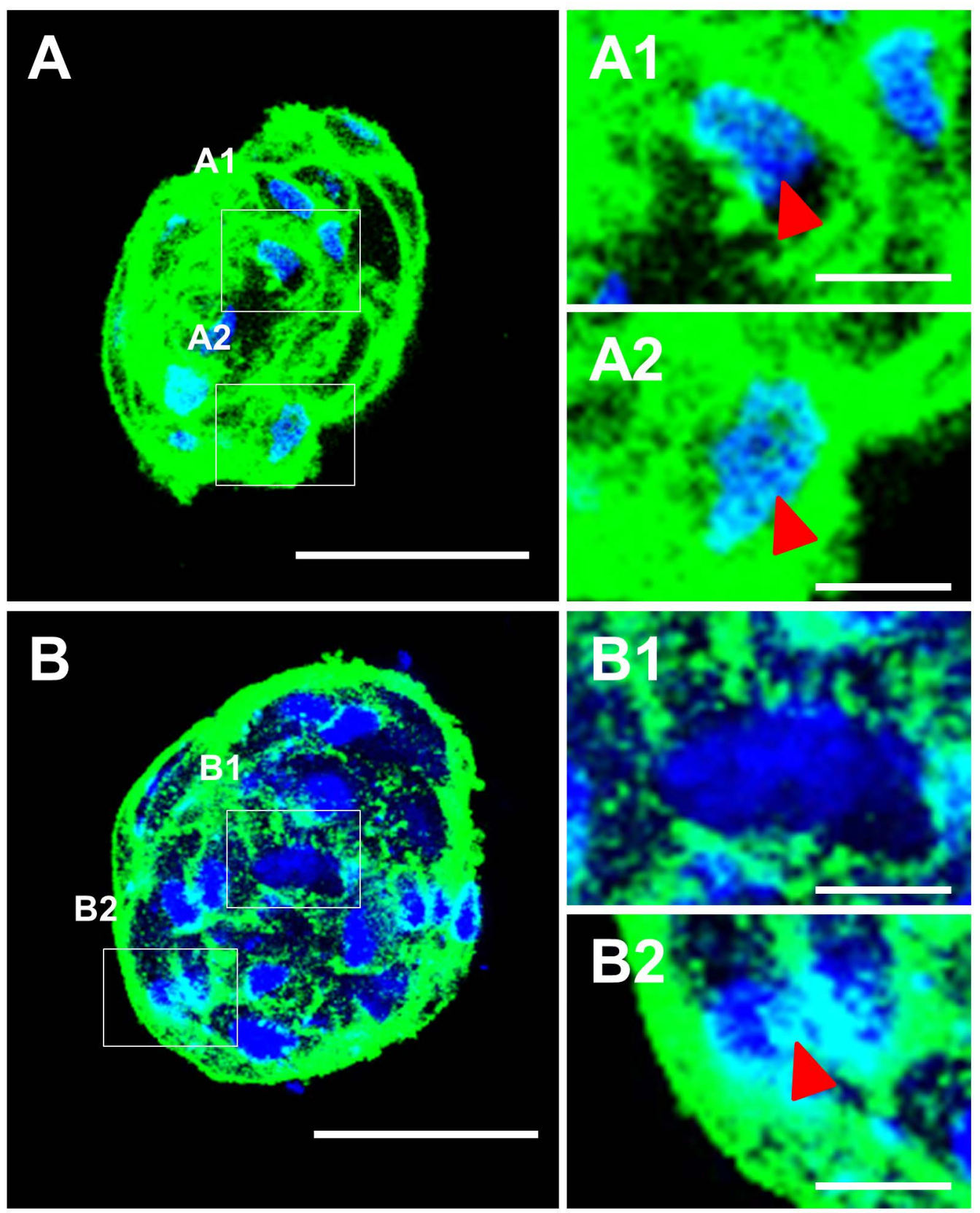
Fig 4

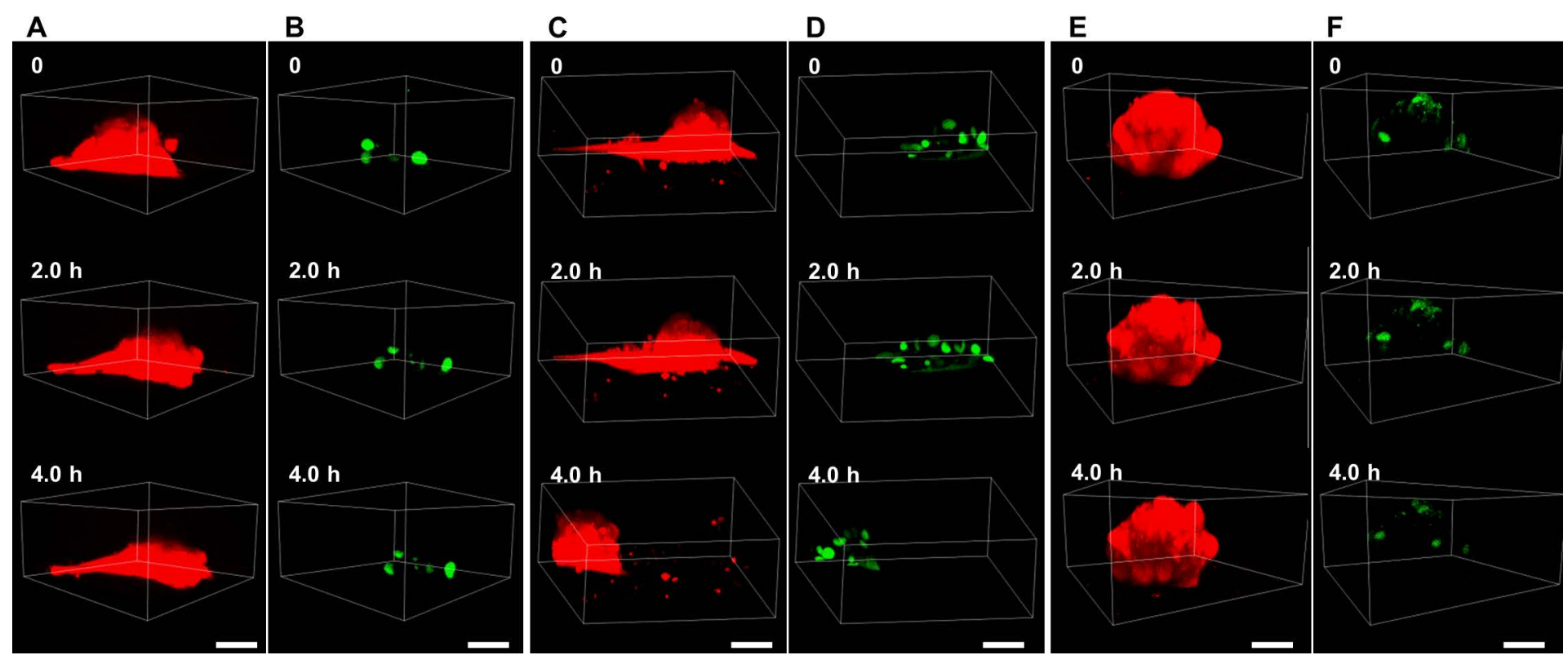

